\title{
Hierarchical sulfur electrodes as a testing platform for understanding the high-loading capability of Li-S batteries
}

\author{
Sheng-Heng Chung, ${ }^{1}$ Chi-Hao Chang, ${ }^{1}$ and Arumugam Manthiram ${ }^{1, *}$ \\ ${ }^{1}$ Materials Science and Engineering Program \& Texas Materials Institute \\ The University of Texas at Austin, Austin, TX 78712, USA
}

Sheng-Heng Chung: shchung@utexas.edu Chi-Hao Chang: chihaochang@utmail.utexas.edu Arumugam Manthiram: manth@austin.utexas.edu

\begin{abstract}
Lithium-sulfur ( $\mathrm{Li}-\mathrm{S})$ batteries are considered as an attractive electrochemical energy storage system due to the high theoretical capacity of sulfur $\left(1,675 \mathrm{~mA} \mathrm{~h} \mathrm{~g}^{-1}\right)$. However, highloading sulfur cathodes would need to be employed for the Li-S cells to be practical, but the resulting poor cell cyclability and severe electrode degradation hamper their development. Here, we present a hierarchical sulfur cathode as a testing platform for understanding the high-loading capability of Li-S batteries. The hierarchical cathode presents good electrochemical utilization of above $70 \%$, stable cyclability for $500-1,000$ cycles, and high sulfur loadings of $4.2-10.0 \mathrm{mg} \mathrm{cm}^{-2}$. The exploration of the activation and the polysulfideretention processes of the high-loading cathodes illustrates that the electrochemical stability mainly results from the stabilization of dissolved polysulfides within the cathode region as the electrochemically active catholyte. Therefore, the utilization of stabilized polysulfide migration might be a meaningful opportunity for designing high-loading cathodes and further improving their electrochemical stability and long-term cyclability.
\end{abstract}

Keywords: lithium-sulfur batteries, structural electrodes, cell configuration, sulfur loading

*To whom correspondence should be addressed

Phone: 512-471-1791. Fax: 512-475-8482. E-mail: manth@austin.utexas.edu (A. Manthiram) 


\section{Introduction}

Lithium-sulfur (Li-S) electrochemical cells currently remain as one of the most promising electrochemical energy storage systems due to their high energy density. Given its high theoretical capacity $\left(1,675 \mathrm{~mA} \mathrm{~h} \mathrm{~g}^{-1}\right)$, great abundance, and low production cost, there is huge interest in engineering the sulfur cathodes into a reality. Thus, success in developing sulfur as an advanced conversion-reaction electrode could push Li-S cells into commercialization [1-5]. However, this procedure is challenged by several inhibitive properties that occur inherently within the sulfur cathode [1-3]. The drawbacks include (i) low electrochemical utilization of sulfur due to the insulating nature of sulfur and the enddischarge products, lithium sulfides; and (ii) electrochemical instability resulting from the severe diffusion and shuttling of redox intermediates, lithium polysulfides [1,6-11]. Uncontrollable polysulfide diffusion further induces the redeposition of a layer of insulating lithium sulfides on the electrode surface and the corrosion of metallic lithium anode [6,7]. The degradation of the sulfur cathode and the lithium-metal anode causes fast capacity fade, low charge efficiency, and even safety concerns [6-8].

To address these scientific and engineering issues, researchers are making great efforts to construct suitable, high-performance sulfur-based cathodes [2,11]. Among them, significant efforts are being especially dedicated to constructing sulfur-carbon nanocomposites. Engineered porous carbon materials, such as micro-/meso-/macroporous carbons, and hierarchical carbon substrates, such as composite multi-dimension carbons, are appealing as active-material containers and to serve as polysulfide holders [12-24]. After encapsulating the active material within the conductive carbon matrix, a close connection between the active material and the conductive network facilitates electron and $\mathrm{Li}^{+}$-ion transfer. Based on this concept, the Li-S technology has received progress in recent years [2,3,12-24].

When it comes to electrode design, the conventional cathode-fabrication process and configuration currently used to make Li-S cells that utilize solid conversion-reaction 
electrodes are directly borrowed from Li-ion batteries that use solid insertion-compound electrodes [12-15,25-28]. In consideration of the totally different battery chemistries, the conventional cathode configuration designed for insertion-reaction cathodes may not be able to make the best use of sulfur cathodes [2,26]. Taking the commonly used sulfur-carbon composite cathode as an example, its fabrication process needs to mix the as-prepared nanocomposites with insulating binders and additional conductive carbon to form an activematerial slurry, and then coat the mixed slurry onto an aluminum-foil current collector in order to constitute a composite cathode. The composite cathode configured in a conventional cathode structure might face three possible risks during Li-S cell cycling. First, the conversion between sulfur and lithium sulfides involves complex $\operatorname{sulfur}_{(\text {solid) }}$-polysulfides(liquid) sulfides $_{\text {(solid) }}$ phase transitions. The rearrangement of the active material could break the close connection between the active material and the conductive carbon host. The electrochemically inaccessible active material gradually enters an inactive area which leads to polarization and cell impedance [1,3]. Second, at intermediate charge and discharge states, both lithium sulfides and sulfur convert to polysulfide intermediates $\left(\operatorname{Li}_{2} \mathrm{~S}_{x}: x=4-8\right)$ that readily dissolve into the ether-based electrolytes currently used in Li-S cells [6,7]. Although ether-based electrolytes are used because of their ability to dissolve polysulfides and thus enhance their reaction kinetics, the dissolved polysulfides would cause severe and irreversible polysulfide diffusion $[4,5,25]$. This implies that the active material trapped within the pockets of nanocomposites might be pulled into liquid electrolytes from their engineered sulfur cages, resulting in irreversible active-material loss and electrode degradation [6,8]. Third, a severe volumetric expansion between sulfur and lithium sulfides of up to $80 \%$ creates stress or even damage to the electrode $[1,2]$. The above factors lead to a degradation of the composite cathodes during cell cycling. [2,25-28]. They also challenge the development of high-loading cathodes and might put a limit on the continuous progresses of sulfur-carbon nanocomposites [25-28]. As a result, about $95 \%$ of the reported Li-S studies utilize low-loading sulfur 
cathodes $\left(<2 \mathrm{mg} \mathrm{cm}^{-2}\right)$ as standard cathodes. However, the battery chemistries and electrochemical characteristics of the low-loading sulfur cathodes are different from the practical high-loading sulfur cathodes [2,10,11]. Moreover, a thick cathode coating has been highlighted as a challenge during cathode preparation and battery operation $[2,11]$.

Advanced carbon engineering has demonstrated that conductive carbon application in Li-S cells benefits sulfur utilization and polysulfide retention if it is configured appropriately $[2,26]$. Thus, the development of structural cathode configurations paves a new way toward effectively utilizing the unique materials chemistry of sulfur and developing corresponding Li-S battery chemistries based on high loading cathodes [12,15,29,30]. Many efforts have focused on suppressing the active-material loss using a porous current collector [30-36] and blocking the active-material diffusion using a free-standing interlayer [35-41] or a multifunctional separator [42-53]. These two approaches either create a polysulfide reservoir to absorb the dissolved polysulfides [31-36] or establish a polysulfide filter to maintain the diffusing polysulfides within the cathode region of the cell [37-53]. By keeping the polysulfide species within the sulfur cathode, both methods improve the cycle stability and electrochemical efficiency due to, respectively, the suppression of polysulfide diffusion and the utilization of polysulfides as a catholyte [31-53]. Several well-designed structural cathodes could even ameliorate the energy density problem that currently arises from lowloading cathodes [26,30-53], which bring us one step closer to the realization of Li-S batteries with high-loading, high-capacity sulfur cathodes. Among these structural cathodes, the hierarchical cathodes show great potential in improving both active-material loading and utilization through a simple cathode engineering [2,3,29]. The hierarchical cathodes create (i) continuous electron pathways and ion-diffusion channels for enhancing the electrochemical utilization of sulfur [19,33], (ii) layered polysulfide traps for suppressing polysulfide diffusion $[33,35,37]$, and (iii) a layer-by-layer cathode configuration for loading with a high amount of active material $[24,28,36,54,55]$. 
In view of the progress with the structural cathodes, we present here a hierarchical cathode as a testing platform for developing high-loading, long-life sulfur cathodes. In order to serve as an electrochemical testing platform, the hierarchical cathodes are controlled to possess a good balance among sulfur loading $\left(4.2 \mathrm{mg} \mathrm{cm}^{-2}\right)$, sulfur content (60 wt.\%), and sulfur mass (4.2 mg cathode $\left.{ }^{-1}\right)$. The hierarchical cathodes demonstrate the enhanced electrochemical stability, exhibiting (i) improved cycle stability with low capacity-fade rates of $0.04-0.08 \%$ per cycle for $500-1000$ cycles and (ii) increasing sulfur loadings of up to $10.0 \mathrm{mg} \mathrm{cm}^{-2}$ with an areal capacity of $8 \mathrm{~mA} \mathrm{~h} \mathrm{~cm}^{-2}$ and a capacity-retention rate of $60 \%$ after 200 cycles. The reason for the hierarchical cathodes to possess high-loading capability and simultaneously display good cyclability is explored by analyzing their activation process and the excellent polysulfide retention brought about by the hierarchical electrode structure. By way of exploring the above-mentioned two electrochemical reactions, we demonstrate that the high-loading cyclability of the high-capacity sulfur cathodes could be enhanced by stabilizing the dissolved polysulfides within the cathode and utilizing them as electrochemically active catholyte to ameliorate the reaction capability of high-loading sulfur cathodes.

\section{Experimental section}

\subsection{Hierarchical cathode fabrication}

The hierarchical cathodes were fabricated by grouting the active-material layer in between a layer of carbon film (NanoTechLabs, Inc.) and the aluminum-foil current collector (Kenkut). The active-material layer contained $80 \mathrm{wt} . \%$ pure sulfur powder, $10 \mathrm{wt} . \%$ Super P carbon (Imerys Graphite \& Carbon), and 10 wt.\% polyvinylidene fluoride binder (Grade No. L\#1120, Kureha). The active-material mixtures were stirred with N-methyl-2-pyrrolidone (Sigma-Aldrich) solvent for two days to form a viscous active-material paste. The mixed active-material paste was first dropped onto the middle of the aluminum-foil current collectors. The carbon-film shield (diameter: $1.13 \mathrm{~cm}$ ) was subsequently placed on top of the 
active-material layer and the aluminum-foil current collectors (diameter: $1.13 \mathrm{~cm}$ ), followed by pressing the edge of the electrode for favorable encapsulation and conservation of the active-material layer. In doing so, the hierarchical cathodes maintain good electrode stabilization during cell cycling. The as-prepared hierarchical cathodes were dried for $24 \mathrm{~h}$ at $50^{\circ} \mathrm{C}$ in an air oven. The resulting hierarchical cathodes have a sulfur loading of $4.2 \mathrm{mg} \mathrm{cm}^{-2}$. The total sulfur mass and content in the cathode are, respectively, $4.2 \mathrm{mg}$ and $60 \mathrm{wt} . \%$. In order to assess the high-loading capability, the hierarchical cathodes with the sulfur loadings of $6.3,8.3$, and $10.0 \mathrm{mg} \mathrm{cm}^{-2}$ were prepared by wrapping additional carbon-film shields and active-material layers. High-loading hierarchical cathodes with the sulfur loadings of 4.2, 6.3, 8.3 , and $10.0 \mathrm{mg} \mathrm{cm}^{-2}$ have, respectively, $1,2,3$, and 4 layers each of carbon-film and activematerial layers. In all cases, the edges of the cathodes were all sealed together so that the active material is confined within the hierarchical cathode. The hierarchical cathodes with increasing sulfur loading had the same sulfur content of $60 \mathrm{wt} . \%$.

The same active-material paste is tape-casted onto an aluminum-foil current collector and dried for $24 \mathrm{~h}$ at $50^{\circ} \mathrm{C}$ in an air oven, followed by roll-pressing and cutting into circular disks. The sulfur loading and content in the conventional cathode disk are, respectively, $2 \mathrm{mg}$ $\mathrm{cm}^{-2}$ and $60 \mathrm{wt} . \%$.

\subsection{Cell assembly}

The hierarchical cathodes were assembled into a CR2032-type coin cell as experimental cells in an argon-filled glove box with a polypropylene separator (Celgard 2500), a lithium-foil anode (Aldrich), a nickel-foam spacer (Pred Materials, Inc.), and a blank electrolyte. In contrast to the experimental cell, the control cells were assembled with conventional cathodes. The blank electrolyte was prepared by dissolving $1.85 \mathrm{M} \mathrm{LiCF}_{3} \mathrm{SO}_{3}$ and $0.1 \mathrm{M} \mathrm{LiNO}_{3}$ co-salts in a 1:1 volume ratio of 1,3-dioxolane (DOL) and 1,2dimethoxyethane (DME). All chemicals for electrolyte preparation were received from Acros 
Organics. The hierarchical cathodes were also assembled into a pouch cell with a polypropylene separator, a lithium-foil anode, and a blank electrolyte. The hierarchical cathodes and lithium anodes were connected to, respectively, an aluminum tab and a nickel tab. Pouch cells were sealed with an aluminum soft packaging film. The assembled coin and pouch cells were allowed to rest for $30 \mathrm{~min}$ at $25^{\circ} \mathrm{C}$ before electrochemical measurements.

\subsection{Multi-functional separator fabrication}

A polyethylene glycol (PEG)-supported microporous carbon (MPC)-coated separator (C-coated separator) was coupled with the hierarchical cathode as an integrated cathode configuration for studying the long-term cycle stability. The C-coated separators were prepared as reported in our previous work [43]. The functional MPC/PEG coating that contained 80 wt.\% MPC (CABOT) and 20 wt.\% PEG (molecular weight $=300 \mathrm{~g} \mathrm{~mol}^{-1}$, Aldrich) was applied onto a Celgard 2500 separator by the tape-casting method. The values of the areal mass of MPC/PEG coating and the separator were, respectively, 0.1 and $1.0 \mathrm{mg} \mathrm{cm}^{-2}$. Due to the light-weight MPC/PEG coating, the integrated cathode configuration maintains a high sulfur content of 59 wt.\% even with the inclusion of the MPC/PEG coating on the cathode side of the separator.

\subsection{Polysulfide-trap cell fabrication}

A polysulfide-trap cell was assembled for analyzing the polysulfide-retention process. The polysulfide-trap cell was configured with a cathode, the first layer of a separator, a carbon-film layer (as the polysulfide trap), the second layer of a separator, and a lithium-foil anode. A reference polysulfide trap was inserted in a control cell using a conventional sulfur cathode and then cycled for 100 cycles. As a comparative analysis, experimental polysulfide traps, on the other hand, were equipped in cells employing the hierarchical cathodes with increasing sulfur loading from 4.2 to $10.0 \mathrm{mg} \mathrm{cm}^{-2}$ and cycled for 200 cycles. 


\subsection{Materials characterization}

Microstructural and elemental analyses were inspected by using a field emission scanning electron microscope (FE-SEM, Quanta 650, FEI) with an energy dispersive X-ray spectrometer (EDX). The cycled cathodes and polysulfide traps were retrieved from cells, rinsed with a 1:1 volume ratio of DOL/DME solution for 5 min, wiped with Kimwipes, and transported into an argon-filled sealed vessel. The sample preparation was conducted inside an argon-filled glove box $30 \mathrm{~min}$ prior to the analysis. The cycled anodes were directly transported for analysis after being retrieving from cells. Carbon structure was investigated by Raman microscopy performed with a micro-Raman System (Alpha 300 S, WITEC).

\subsection{Electrochemical measurements}

Cyclic voltammetry (CV) measurements were scanned between 1.8 and $2.8 \mathrm{~V}$ at a scan rate of $0.1 \mathrm{mV} \mathrm{s}^{-1}$ with a universal potentiostat (VoltaLab PGZ 402, Radiometer Analytical). Discharge and charge profiles and cyclability data were evaluated under galvanostatic conditions between 1.8 and $2.7 \mathrm{~V}$ with a programmable battery cycler (Arbin Instruments). The cells used for electrochemical analyses were first discharged from OCV to $1.8 \mathrm{~V}$ and then charged to $2.7 \mathrm{~V}$ for a full cycle. The upper-plateau discharge capacity $\left(\mathrm{Q}_{\mathrm{H}}\right)$ and lower-plateau discharge capacity $\left(\mathrm{Q}_{\mathrm{L}}\right)$ were collected from the discharge data points. The calculation of capacity-retention rates was based on the values of the mass of active material and the theoretical capacities $\left(\mathrm{Q}_{\mathrm{H}}: 419 \mathrm{~mA} \mathrm{~h} \mathrm{~g}{ }^{-1}, \mathrm{Q}_{\mathrm{L}}: 1,256 \mathrm{~mA} \mathrm{~h} \mathrm{~g}^{-1}\right.$, and $\mathrm{Q}_{\text {total }}: 1,675 \mathrm{~mA} \mathrm{~h} \mathrm{~g}^{-1}$.

\section{Results and Discussion}

\subsection{Configuration and morphology of the hierarchical cathodes}

Figures 1a - 1c summarize the physical and chemical analyses of the carbon-film shield. In Figure 1a, the SEM observation indicates that the carbon-film shield has a woven carbon nanotube (CNT) and carbon nanofiber (CNF) network. The curved CNTs are firmly 
entangled with the CNF skeleton, which creates a conductive and porous network. In Figure $1 \mathrm{~b}$, the Raman spectrum differentiates two prominent peaks at $\sim 1,579$ ( $\mathrm{G}$ band) and $\sim 1,355$ $\mathrm{cm}^{-1}$ ( $\mathrm{D}$ band). The G-band and D-band peaks are caused by, respectively, the in-plane vibration of the $\mathrm{SP}^{2}$ carbon and the phonon scattering at defect sites. A high intensity ratio of the $\mathrm{G}$ band to the $\mathrm{D}$ band indicates a high graphitization level and suggests fast electron transfer $[19,20]$. The porous network and conductive framework are key factors for carbonfilm shields to improve the polysulfide retention and electrochemical utilization of the hierarchical cathode. In addition, the carbon-film shields contribute no additional capacity in the Li/carbon film control cell (Figure 1c), which indicates their good electrochemical stability as being applied in the hierarchical cathode. The electrochemically stable carbon film is covered onto a layer of active-material coating as a carbon-film shield to form the hierarchical cathode. The configuration of the hierarchical cathode is shown in Figure 1d.

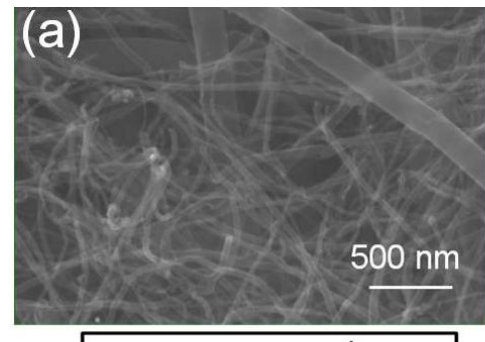

\section{(d)}
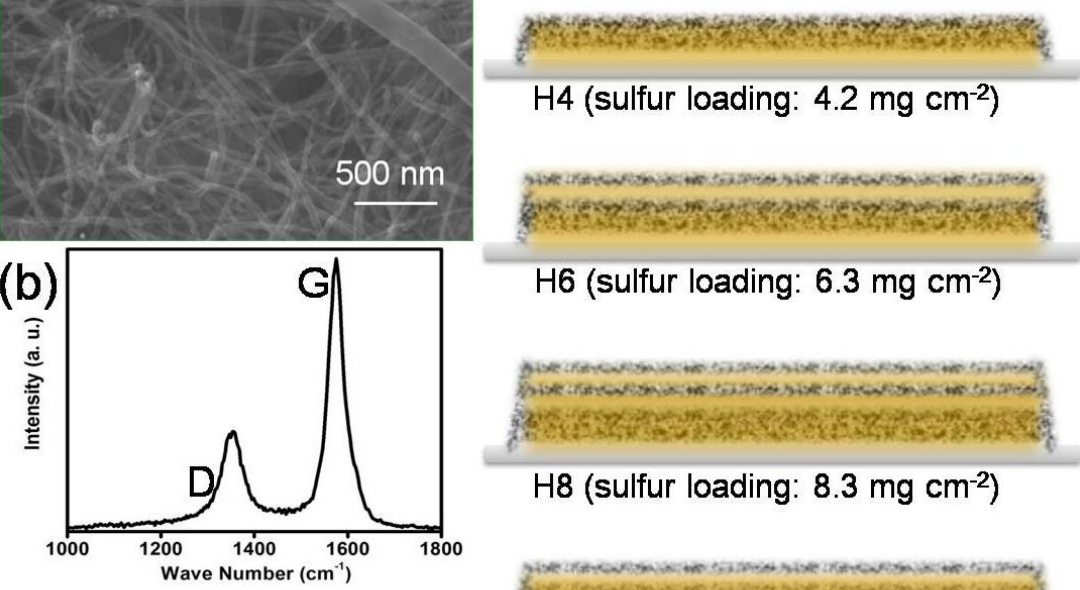

H6 (sulfur loading: $6.3 \mathrm{mg} \mathrm{cm}^{-2}$ )
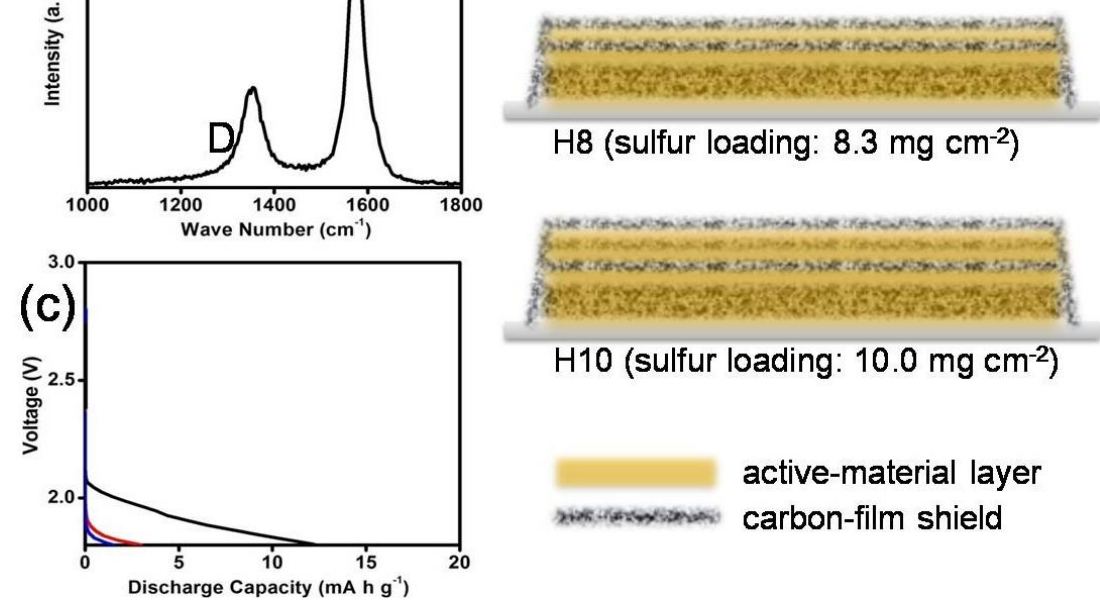

Figure 1 Physical and chemical analysis of the carbon-film shield: (a) SEM, (b) Raman spectrum, and (c) electrochemical analysis. (d) Electrode configuration of the hierarchical cathodes with increasing sulfur loadings of $4.2,6.3,8.3$, and $10.0 \mathrm{mg} \mathrm{cm}^{-2}$. 


\subsection{Stabilization of hierarchical cathodes}

Figure 2a shows the SEM image and the corresponding EDX analysis of an uncycled hierarchical cathode. Under microstructural observation, the carbon-film shield that is covered on the active-material layers shows a well-maintained CNT-CNF network, as compared to that of the pristine carbon films. Preserved conductive CNT-CNF networks are also reflected in the strong elemental carbon signals detected in the EDX mapping results. SEM/EDX results evidence that the carbon-film shield is initially free of the redox products. In order to strengthen this statement, Figure $2 b$ shows the SEM/EDX inspection inside the hierarchical cathode construction. The sample used in the inside SEM/EDX analysis was prepared by peeling the aluminum-foil current collector from an uncycled hierarchical cathode and carefully removing parts of the active-material layer with a razor blade before the SEM observation. The observed results provide solid evidence that the carbon-film shield is initially free of sulfur, which is in agreement with Figure 2a. Thus, the carbon-film shield brings abundant porous nanospaces that could channel liquid electrolytes to boost the reaction kinetics of polysulfides [4-6] and activate the active-material layer [37-39]. On the other hand, the carbon-film shield was free of polysulfides initially, which implies that it would create difficulty for dissolved polysulfides to diffuse out from its tortuous CNT-CNF network or from the cathode region upon cycling and thereby increase the capacity retention [37-49].

In order to demonstrate the ability of the hierarchical cathode in stabilizing its activematerial layer, SEM/EDX analysis was performed on a cycled hierarchical cathode (at $2.7 \mathrm{~V}$ $v s . \mathrm{Li}^{+} / \mathrm{Li}^{0}, 500$ cycles) from various perspectives. Figures $2 \mathrm{c}$ and $2 \mathrm{~d}$ show the outer-layer and inner-layer microstructure inspection of the carbon-film shield from the cycled hierarchical cathode. The outer-layer observation shows that the cycled carbon-film shield maintains its original CNT-CNF network, showing a smooth exterior with no surface coverings. Although no agglomerated active-material deposits are seen on the carbon-film shield, elemental sulfur signals are distinguishable. This suggests that the active material is embedded within the 
hierarchical cathode during electrochemical cycling. As a follow-up microstructural inspection, Figure $2 d$ shows the inner-layer observation of the carbon-film shield. The SEM sample used in the inner-layer observation was prepared by removing the outer-layer CNTCNFs so that the inner-layer of the carbon-film shield became detectable. The inner-layer SEM/EDX inspection exhibits much stronger sulfur signals as compared to those detected in the outer-layer inspection. The intensity difference of the elemental sulfur signals demonstrates that the active-material layer is ensconced by the carbon-film shield and is, therefore, encapsulated within the hierarchical cathode.

Because of the suppressed polysulfide diffusion, as expected, the SEM observations display no insoluble $\mathrm{Li}_{2} \mathrm{~S}_{2} / \mathrm{Li}_{2} \mathrm{~S}$ deposits on the hierarchical cathode. Unfavorable $\mathrm{Li}_{2} \mathrm{~S}_{2} / \mathrm{Li}_{2} \mathrm{~S}$ deposits caused by the redeposition of the escaping polysulfides gradually build up insulting layers on the electrode surface and form inactive areas $[7,41,45]$. In addition, there are also no evident active-material buildups blocking the porous spaces of the hierarchical cathodes as a result of the superior conductivity of the hierarchical electrode. The electron pathways and electrolyte channels facilitate fast $\mathrm{Li}^{+}$-ion and electron accesses so as to ensure successive electrochemical redox reactions during repeated cycling, thereby avoiding the accumulation of redox by-products from unsuccessful sulfur-polysulfides-sulfides conversions [6,44].

Therefore, the SEM/EDX analysis of the carbon-film shield before and after cycling summarized in Figure 2 suggests that the hierarchical cathode configuration improves activematerial encapsulation, polysulfide stabilization, and electrochemical-reaction capability. 

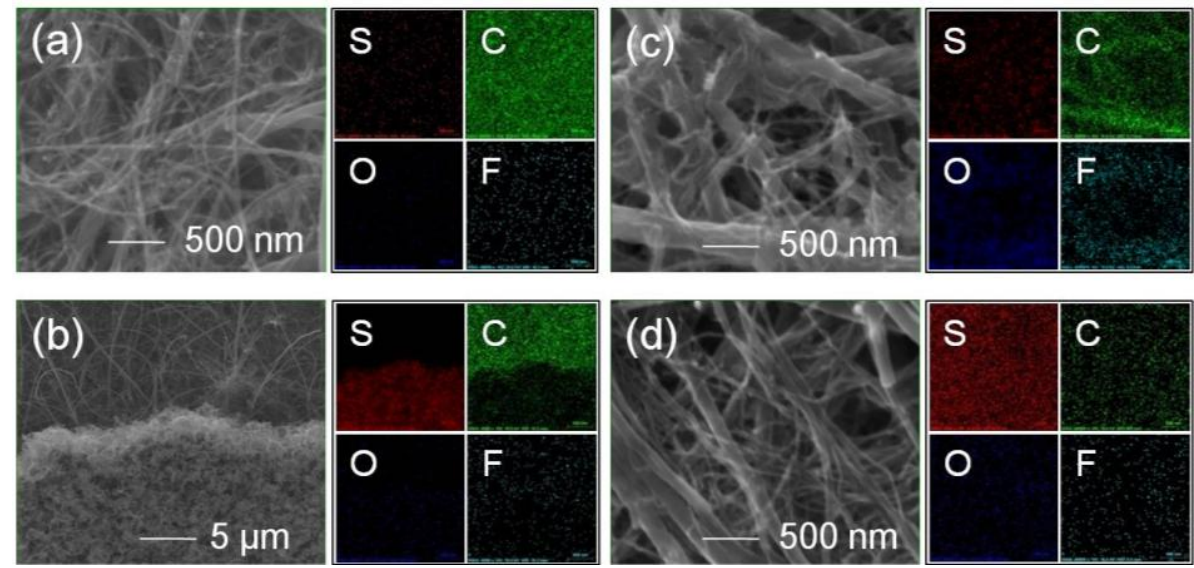

Figure 2 SEM/EDX inspection of (a) uncycled hierarchical cathode and (b) inside observation of (a). SEM/EDX inspection of (c) cycled hierarchical cathode and (d) inner-layer observation of (c).

\subsection{Electrode stabilization}

The abovementioned three improvements resulting from the cathode design aim to stabilize the sulfur cathode and reduce the migration of polysulfides from the cathode side to the anode side of the cells. Thus, SEM/EDX inspection was conducted on the cycled cathode and anode active materials for the purpose of exploring the electrode stabilization of an experimental cell and, in contrast, the electrode degradation of a control cell

Figure 3a shows the cycled active-material layer at a fully charged state (at $2.7 \mathrm{~V} v s$. $\mathrm{Li}^{+} / \mathrm{Li}^{0}, 500$ cycles) in an experimental cell, which employs the hierarchical cathode, where strong sulfur signals are uniformly distributed within the carbon matrix. The close connection between sulfur species and conductive carbon results from the hierarchical cathode that stores the dissolved polysulfides within the cathode region so as to encapsulate the active material within the structural cathode architecture. Moreover, the stabilized polysulfides are electrochemically active and could assist in (i) the activation of the insulating active material during repeated cycling and (ii) the re-activation of the accumulated redox by-products from incomplete electrochemical conversions $[4,5,25]$. As a result of excellent polysulfide retention and smooth redox reactions, there is almost no trace of $\mathrm{Li}_{2} \mathrm{~S}_{2} / \mathrm{Li}_{2} \mathrm{~S}$ agglomerates and cathode delamination during long-term cycling. In contrast, surface morphology of a cycled 
conventional cathode (at $2.7 \mathrm{~V} v s . \mathrm{Li}^{+} / \mathrm{Li}^{0}, 100$ cycles) used in a control cell is characterized by a layer of insoluble $\mathrm{Li}_{2} \mathrm{~S}_{2} / \mathrm{Li}_{2} \mathrm{~S}$ deposits (Figure 3b), a result of the redeposition of the escaping polysulfides on the cathode surface $[6,7]$. The insoluble deposits block the $\mathrm{Li}^{+}$-ion and electron pathways and lead to a low electrochemical utilization of conventional sulfur cathodes $[7,41]$.

On the other side, the cycled lithium-metal anode that was used in the experimental cell (Figure 3c) exhibits a relatively smooth surface and weak sulfur signals. SEM/EDX inspection demonstrates suppressed electrode corrosion and polysulfide migration as well as reduced $\mathrm{Li}_{2} \mathrm{~S}_{2} / \mathrm{Li}_{2} \mathrm{~S}$ precipitation on the cycled anode $[24,44,56,57]$. In contrast, the cell using a conventional cathode without proper protection from a carbon-film shield shows typical lithium-anode degradation. It is evident that a rough lithium surface and high elemental sulfur signals are observed in the SEM/EDX inspection (Figure 3d) [56,57]. As a reference, the cycled lithium-metal anodes retrieved from the cells were directly transported for SEM/EDX analysis. The solution-rinsing step was skipped in order to avoid the washout of sulfurcontaining species from the reacted lithium metal and the unwanted chemical reactions between the rinsing solution and lithium.

A comparison of the morphological and elemental analysis in Figure 3 demonstrates that the hierarchical cathodes alleviate severe polysulfide migration from the cathode side to the anode side of cells. Therefore, the hierarchical cathodes and, even more so, their carbonfilm shield exhibit (i) direct cathode optimization by stabilizing the active material within the structural cathode, thereby achieving (ii) indirect anode stabilization by reducing polysulfide corrosion. 

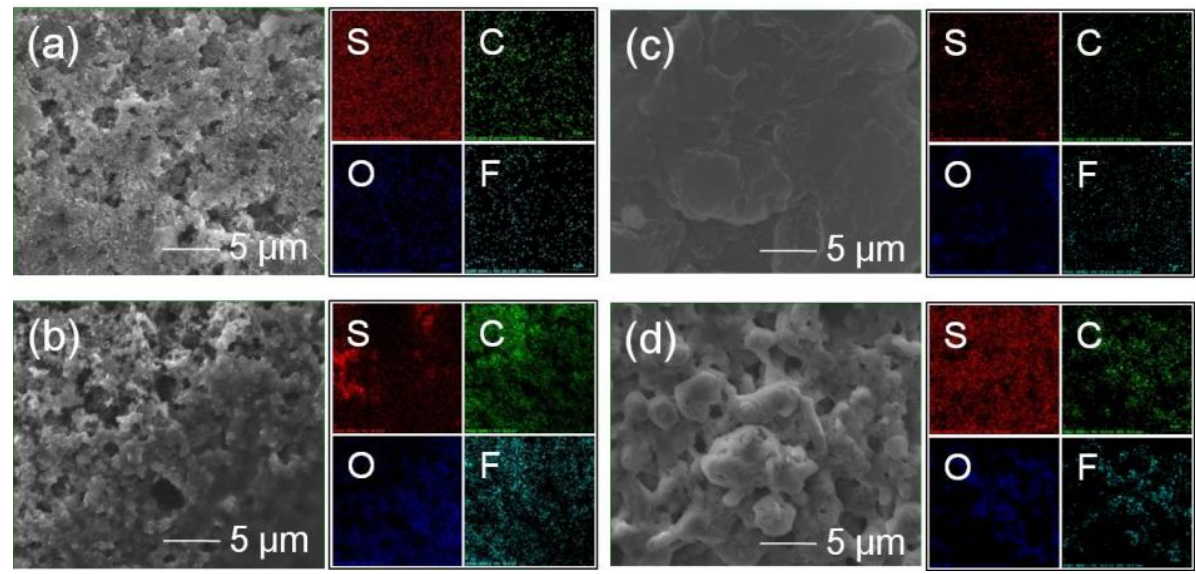

Figure 3 SEM/EDX inspection of the cathode active material of (a) a cycled experimental cell employing the hierarchical cathode and (b) a cycled control cell employing the conventional cathode. SEM/EDX inspection of the anode active material of (c) a cycled experimental cell employing the hierarchical cathode and (d) a cycled control cell employing the conventional cathode.

\subsection{Electrochemical stability}

Figures $4 \mathrm{a}-4 \mathrm{c}$ show the discharge and charge characteristics of the Li-S cells employing the hierarchical cathodes at various cycling rates. In Figure 4d, the cyclic voltammograms $(\mathrm{CV})$ are scanned at $0.1 \mathrm{mV} \mathrm{sec}^{-1}$ in parallel to evaluate electrochemical stability. The two-step redox reactions between $\mathrm{Li}_{2} \mathrm{~S}_{8} / \mathrm{S}$ and $\mathrm{Li}_{2} \mathrm{~S}_{2} / \mathrm{Li}_{2} \mathrm{~S}$ shown in the discharge and charge curves are consistent with the two cathodic peaks and two anodic peaks in the CV scanning [58-63]. The reversible discharge/charge curves and the overlapping CV curves demonstrate improved electrochemical stability $[45,47,50]$.

In Figures $4 \mathrm{a}-4 \mathrm{c}$, the upper-discharge plateau at $2.3 \mathrm{~V}$ indicates the formation of

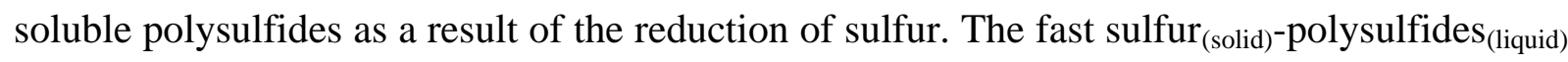
reduction reactions involve the formation, dissolution, and diffusion of polysulfides [1,6,52]. This electrochemical-conversion stage, therefore, indicates the polysulfide-diffusion status $[6,42,46]$. The high utilization rates of the $\mathrm{Q}_{\mathrm{H}}$ attain $93 \%(\mathrm{C} / 10$ rate), $90 \%(\mathrm{C} / 5$ rate), and $60 \%(\mathrm{C} / 2$ rate) (Figure $4 \mathrm{e})$. After 50 cycles, the $\mathrm{Q}_{\mathrm{H}}$ retention rates attain $85+\%$ at various cycling rates. The high utilization and retention rates of $\mathrm{Q}_{\mathrm{H}}$ indicate reduced polysulfide diffusion $[8,44,58-60]$. On the other hand, the lower-discharge plateau at $2.0 \mathrm{~V}$ explains the 
slow transformations from liquid-phase polysulfides to solid-phase sulfides. In consideration of the formation and re-deposition of solid-phase sulfides, this conversion stage and the corresponding $\mathrm{Q}_{\mathrm{L}}$ are reflected in the reduction capability of the corresponding cells and their capability in avoiding the formation of agglomerated active-material buildups in the electrode $[8,44,46]$. The hierarchical cathodes exhibit a high $\mathrm{Q}_{\mathrm{L}}$ retention rate of $75+\%$ at various cycling rates (Figure 4f), indicating a thorough reduction of the trapped active material and excellent electrochemical reversibility $[2,8,44]$. The favorable features of $\mathrm{Q}_{\mathrm{H}}$ and $\mathrm{Q}_{\mathrm{L}}$ analyses work in concert with the microstructural observation and explain why there is no surface passivation and accumulation of redox by-products, respectively, from severe polysulfide diffusion and unsuccessful sulfur-polysulfides-sulfides conversions $[28,44]$.

During cell charge, two continuous charge plateaus at $2.3-2.4 \mathrm{~V}$ represent reverse oxidation reactions [1,61-63]. The cells exhibit a high Coulombic efficiency of above 98\% because of the use of the hierarchical cathode and $\mathrm{LiNO}_{3}$ co-salts. The hierarchical cathode indirectly stabilizes lithium-metal anode via a suppression of polysulfide diffusion from the cathode $[39,44]$. The $\mathrm{LiNO}_{3}$ co-salt additives directly protect lithium-metal anodes via the formation of a passivation $\mathrm{NO}_{\mathrm{x}}$-film on the anode $[28,53]$. 
(a)

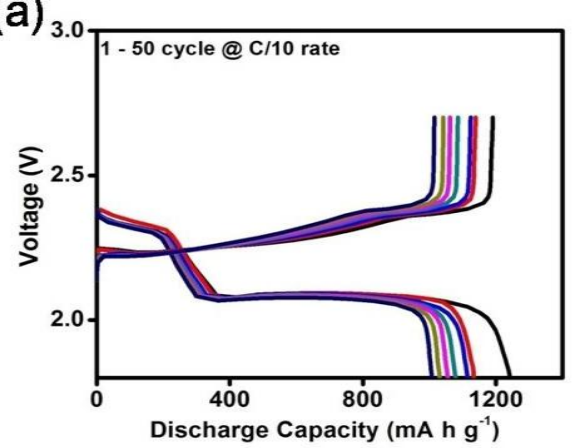

(b)

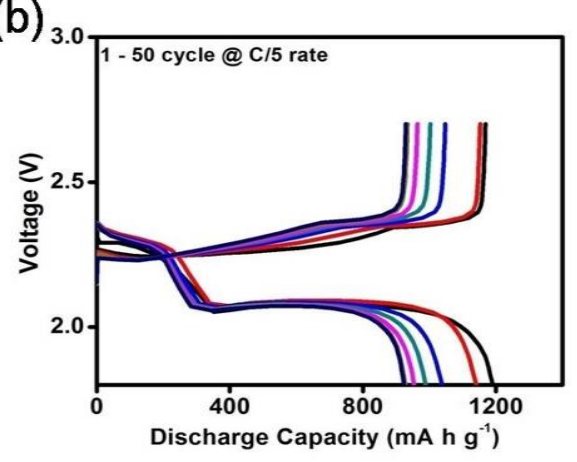

(c)

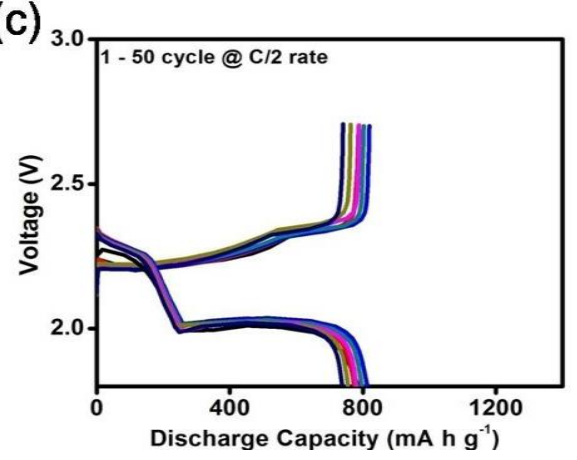

(d)

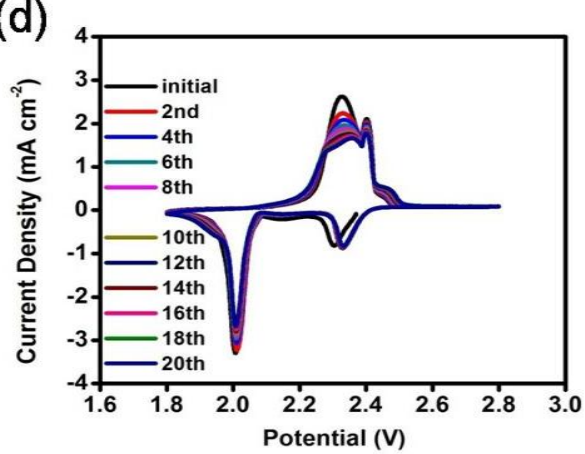

(e)

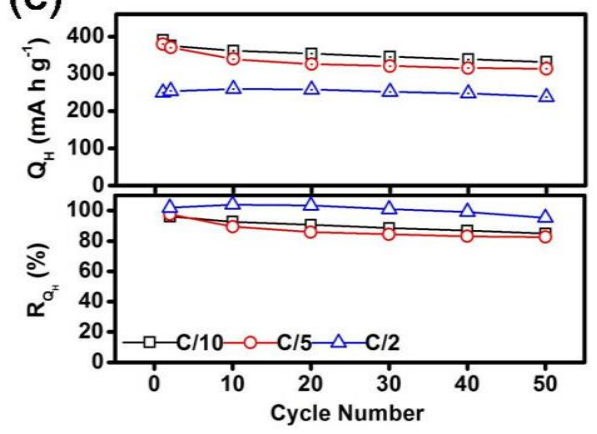

(f)

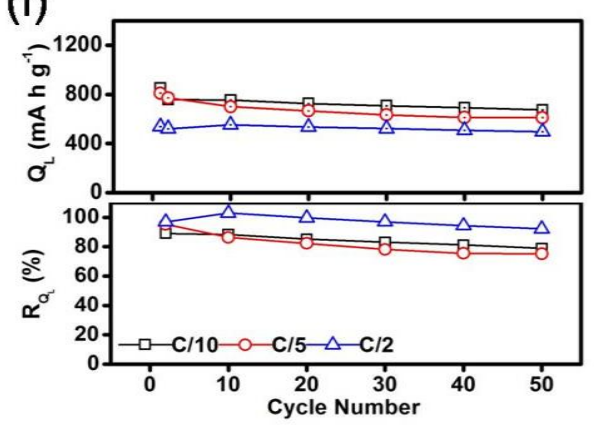

Figure 4 Discharge and charge profiles of Li-S cells employing the hierarchical cathodes at (a) $\mathrm{C} / 10$, (b) $\mathrm{C} / 5$, and (c) $\mathrm{C} / 2$ rates. (d) $\mathrm{CV}$ scan curves. (e) $\mathrm{Q}_{\mathrm{H}}$ and (f) $\mathrm{Q}_{\mathrm{L}}$ analysis.

Excellent electrochemical utilization and stability allow the hierarchical cathodes to attain high discharge capacity and long-term cyclability for 500 cycles at various cycling rates (Figure 5a). Peak discharge capacities approach 1,242, 1,190, and $816 \mathrm{~mA} \mathrm{~h} \mathrm{~g}{ }^{-1}$ at, respectively, $\mathrm{C} / 10, \mathrm{C} / 5$, and $\mathrm{C} / 2$ rates. An average electrochemical utilization of sulfur reaches $65 \%$. After cycling for 500 cycles at various cycling rates, the corresponding reversible capacities are 608,502 , and $374 \mathrm{~mA} \mathrm{~h} \mathrm{~g}^{-1}$. The capacity-fade rates are as low as $0.05-0.08 \%$ per cycle. These features provide electrochemical evidence that the hierarchical cathodes simultaneously exhibit good cell performance and achieve high sulfur loading. 
The electrochemical characteristics characterized in Figures 4 and 5a confirm improved electrochemical reversibility and high-loading capability in the hierarchical cathodes. Such stable cyclability results from adopting the materials chemistry of sulfur: the stabilization of dissolved polysulfides within the cathode and their utilization as the electrochemically active catholyte [58-60]. Although polysulfide dissolution and migration should be inhibited in the case of composite cathodes [12-24], these two processes are allowed in hierarchical cathodes and have two brand-new functions. First, the dissolved polysulfides are channeled within the CNT-CNF network and remain at electrochemically stable positions. This in-situ rearrangement brings benefits in enhancing the electrochemical reversibility of the hierarchical cathodes [37-39]. Second, the dissolved polysulfides are electrochemically active and function as catholytes within the hierarchical cathodes [61-63]. They assist with the activation of the bulk sulfur clusters that are initially buried inside the active-material layer $[62,63]$. As the embedded sulfur is uniformly exposed to the electrolyte, the high-loading hierarchical cathodes exhibit a high electrochemical utilization. Therefore, the utilization of the unique characteristics of sulfur, i.e., polysulfide dissolution and migration, might also facilitate progress on high-loading cathodes.

An extended study integrates the hierarchical cathodes with a multi-functional separator (polyethylene glycol (PEG)-supported microporous carbon (MPC)-coated separator: C-coated separator) [43] to demonstrate the benefits of comprehensive cathode engineering on boosting the overall cell performance (Figure 5b). Functional MPC/PEG coating offers additional conductive pathways onto the hierarchical cathodes. Improved cathode conductivity enhances the electrochemical utilization of active-material layers so that the peak discharge capacities further increase to $1,261,1,240$, and $1,177 \mathrm{~mA} \mathrm{~h} \mathrm{~g}^{-1}$ at the higher cycling rates of, respectively, $\mathrm{C} / 5, \mathrm{C} / 2$, and $1 \mathrm{C}$. Besides enhancing the electrochemical utilization and rate capability, the MPC/PEG coating also strengthens the polysulfide-trapping capability. Improved capacity retention is reflected in the high reversible capacities of 601, 588, and 576 
$\mathrm{mA} \mathrm{h} \mathrm{g}^{-1}$ after 500 cycles. The attractive cycle stability results in a low capacity-fade rate of $0.07 \%$ per cycle. Moreover, the experimental cells using a hierarchical cathode and a multifunctional separator that are cycled at $\mathrm{C} / 5$ rate are utilized as a demonstration for an ultralong-term cycling test. The result shows that high-loading cathodes are able to attain a low capacity-fade rate of $0.04 \%$ per cycle and a good Coulombic efficiency of $98+\%$ for over 1,000 cycles. This extended study demonstrates superior battery performances, such as: (i) enhanced electrochemical utilization of sulfur up to 75,74 , and $70 \%$ at, respectively, $\mathrm{C} / 5$, $\mathrm{C} / 2$, and $1 \mathrm{C}$ rates, and (ii) reduced capacity fade rates of $0.04-0.07 \%$ per cycle at high cycling rates.
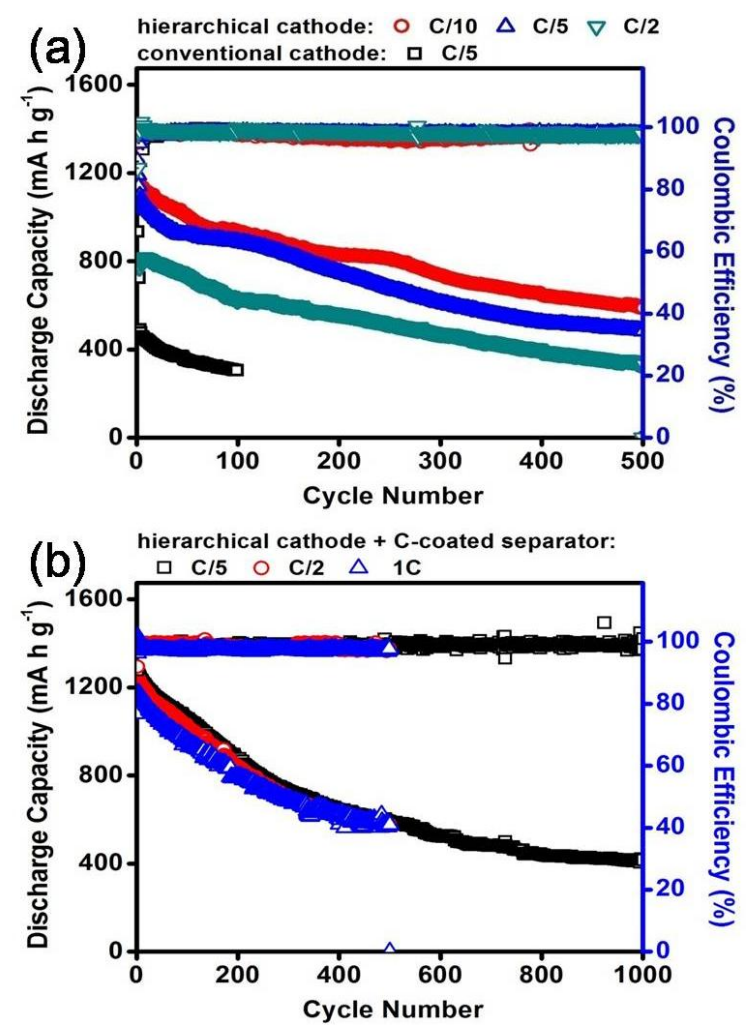

Figure 5 Electrochemical characteristics: Li-S cells employing (a) the hierarchical cathodes at various cycling rates and (b) the hierarchical cathodes and the $\mathrm{C}$-coated separators at various cycling rates.

\subsection{Battery performances}

The use of hierarchical cathodes in Li-S cells demonstrates the potential for optimizing the electrochemical characteristics. In order to identify their capability in accommodating 
high sulfur loadings, active-material layers incorporated into the hierarchical cathodes were loaded with a higher amount of active material from $4.2 \mathrm{mg} \mathrm{cm}^{-2}$ to $6.3,8.3$, and $10.0 \mathrm{mg} \mathrm{cm}^{-2}$.

Figures $6 a-6 c$ show the battery performances of the cells that employ the hierarchical cathodes with increasing sulfur loadings. The initial discharge capacities of the hierarchical cathodes with increasing sulfur loadings of 4.2, 6.3, 8.3, and $10.0 \mathrm{mg} \mathrm{cm}^{-2}$ are, respectively, $1,242,520,508$, and $506 \mathrm{~mA} \mathrm{~h} \mathrm{~g}^{-1}$. It shows a decrease in the initial discharge capacity with increasing sulfur loading and an activation process that is marked in blue is found in the cells with sulfur loadings of above $6.3 \mathrm{mg} \mathrm{cm}^{-2}$. These phenomena are associated with an increasing sulfur loading that (i) increases the number of insulating active-material layers and (ii) generates bulk sulfur clusters inside the active-material layers. These features increase cathode resistance and reduce electrochemical utilization $[2,26]$. By way of utilizing the hierarchical cathode configuration, insulating active-material layers and bulk sulfur clusters could be activated by catholytes, the dissolved polysulfides, when the diffusing polysulfides migrate within the hierarchical cathode [37-39,61]. Thus, after 5 - 15 cycles, the hierarchical cathodes with sulfur loadings of $6.3,8.3$, and $10.0 \mathrm{mg} \mathrm{cm}^{-2}$ approach their peak capacities of, respectively, 1,201, 935, and $755 \mathrm{~mA} \mathrm{~h}^{-1}$. The corresponding peak areal capacities attain $5-$ $8 \mathrm{~mA} \mathrm{~h} \mathrm{~cm}{ }^{-2}$. After 200 cycles, the reversible areal capacity has an average value of $4.2 \mathrm{~mA} \mathrm{~h}$ $\mathrm{cm}^{-2}$. The improved areal capacity is almost two times higher than the value of commercially available lithium-ion batteries [17,30]. The high-loading hierarchical cathode was further incorporated into a large-sized pouch cell. The cathode has a sulfur loading of $10.0 \mathrm{mg} \mathrm{cm}{ }^{-2}$ and a sulfur mass of up to $50 \mathrm{mg}$. Figure $6 \mathrm{c}$ shows that the battery performances of the pouch cell and a coin cell are about the same. The hierarchical cathode provides the pouch cell with a stable discharge capacity of $800 \mathrm{~mA} \mathrm{~h} \mathrm{~g}^{-1}$. The peak discharge capacity and areal capacity attain, respectively, $815 \mathrm{~mA} \mathrm{~h} \mathrm{~g}^{-1}$ and $8.15 \mathrm{~mA} \mathrm{~h} \mathrm{~cm}^{-2}$.

Figures $6 \mathrm{~d}-6 \mathrm{~g}$ display the SEM/EDX analysis of the cycled high-loading hierarchical cathodes. The SEM images of the cycled hierarchical cathodes do not show obvious sulfur 
buildups on their surface, nor within their porous network. As a result, the corresponding EDX mapping results reflect in weak sulfur signals. These features coupled with the improved battery performances illustrate that the application of the hierarchical electrode configuration in building high-loading sulfur cathodes could suppress the severe polysulfide diffusion during battery cycling.
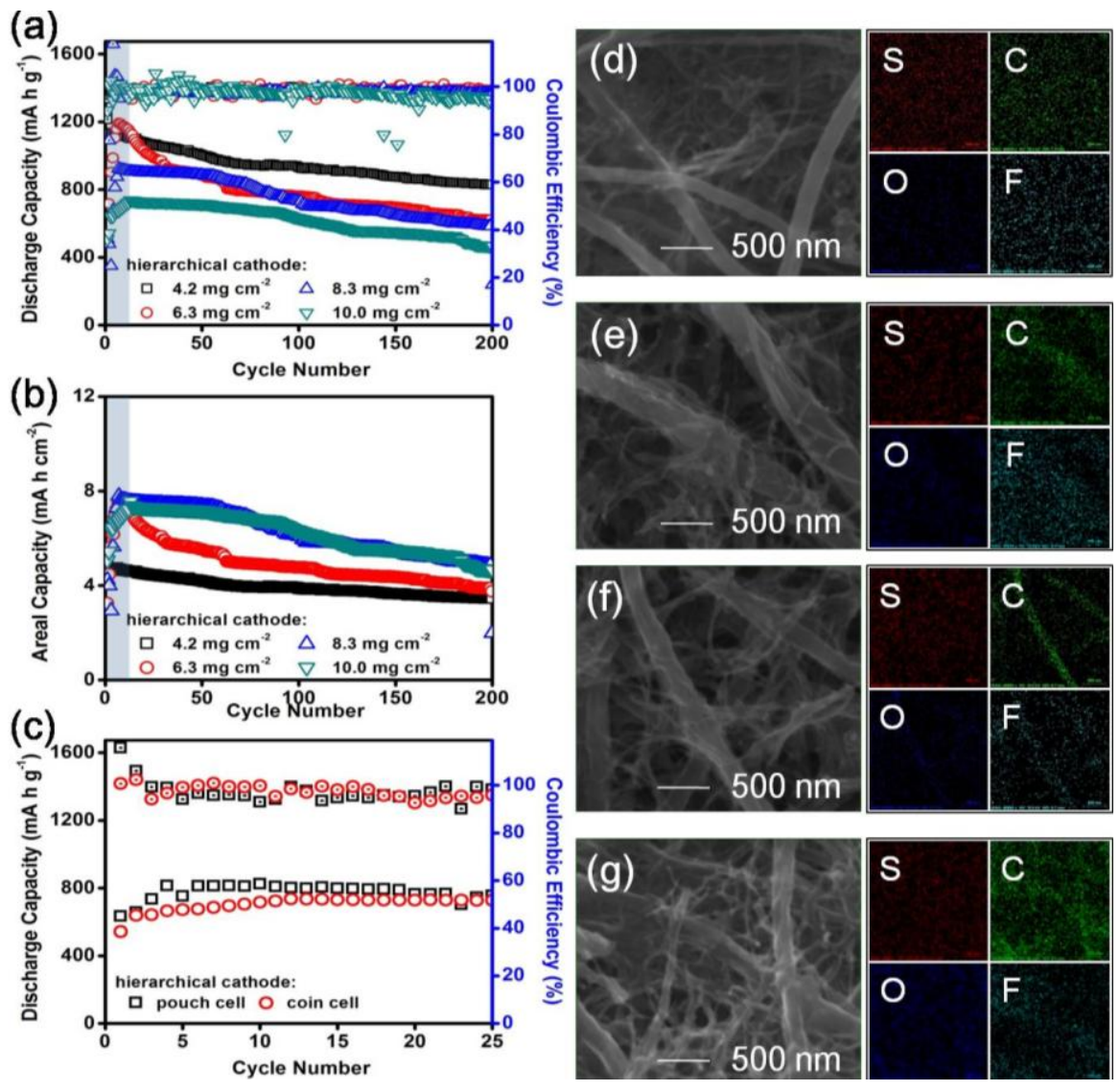

Figure 6 Battery performances: (a) Li-S cells employing the hierarchical cathodes with various sulfur loadings from 4.2 to $10.0 \mathrm{mg} \mathrm{cm}^{-2}$ and (b) the corresponding areal capacity $\left(\mathrm{mA} \mathrm{h} \mathrm{cm}{ }^{-2}\right.$ ) of (a). (c) Battery performances of the Li-S cells employing the hierarchical cathodes with a sulfur loading of $10.0 \mathrm{mg} \mathrm{cm}^{-2}$ in a pouch cell and in a coin cell. $(\mathrm{d}-\mathrm{g})$ SEM/EDX inspection of the cycled hierarchical cathodes with sulfur loadings of 4.2, 6.3, 8.3, and $10.0 \mathrm{mg} \mathrm{cm}^{-2}$.

\subsection{Activation process of the high-loading cathodes}

In consideration of the increasing capacity evident in the initial several cycles, it is imperative to investigate the activation process of the active-material layers that contain a high amount of sulfur. According to the above analysis, hierarchical cathodes with a sulfur loading of $6.3 \mathrm{mg} \mathrm{cm}^{-2}$ were sampled in the following investigation. In Figures $7 \mathrm{a}-7 \mathrm{c}$, cross- 
sectional SEM inspections of the hierarchical cathodes at various cycles $(0,5$, and 200 cycles) demonstrate the in-situ rearrangement of the active-material layers step-by-step.

The uncycled cathode shows the hierarchical electrode configuration that consists of carbon-film shields and active-material layers. According to the electrochemical analysis described above, an initial increase in battery capacity occurs during the initial 10 cycles. As a result, cells are opened for the inspection of the reacted cathodes after the fifth cycle at the charged state (at $2.7 \mathrm{~V} v s . \mathrm{Li}^{+} / \mathrm{Li}^{0}$ ). It is evident that sulfur-containing species start to diffuse out from the active-material layers and are absorbed by the conductive carbon-film shields, as a result of the stabilized polysulfide migration. After 200 cycles, the diffusing polysulfides are stabilized within the hierarchical electrode and the elemental sulfur signals are, therefore, uniformly distributed in the conductive matrix.

Microstructural analysis shows evidence that the rearranged active material gradually enters the intricate three-phase architecture among the active material, conductive carbon-film shield, and electrolytes. Smooth electron and electrolyte accesses promote the redox-reaction capability of cells. Such optimal rearrangement of the active material results from the beneficial polysulfide diffusion: the stable polysulfide diffusion within the cathode region. Furthermore, the dissolved polysulfides are electrochemically active catholytes which assist in activating the active-material layers and subsequently raising their electrochemical utilization and reaction capability. Thus, cells receive additional capacity from the inner sulfur clusters of high-loading cathodes in the initial several cycles. In the following electrochemical reactions, the active-material rearrangement that is stabilized within the cathode region could reduce capacity fade and increase capacity retention during long-term cycling. Therefore, the development of high-loading sulfur cathodes may depend more on the utilization of the materials chemistry of sulfur rather than restricting it as conventional concepts have done. 


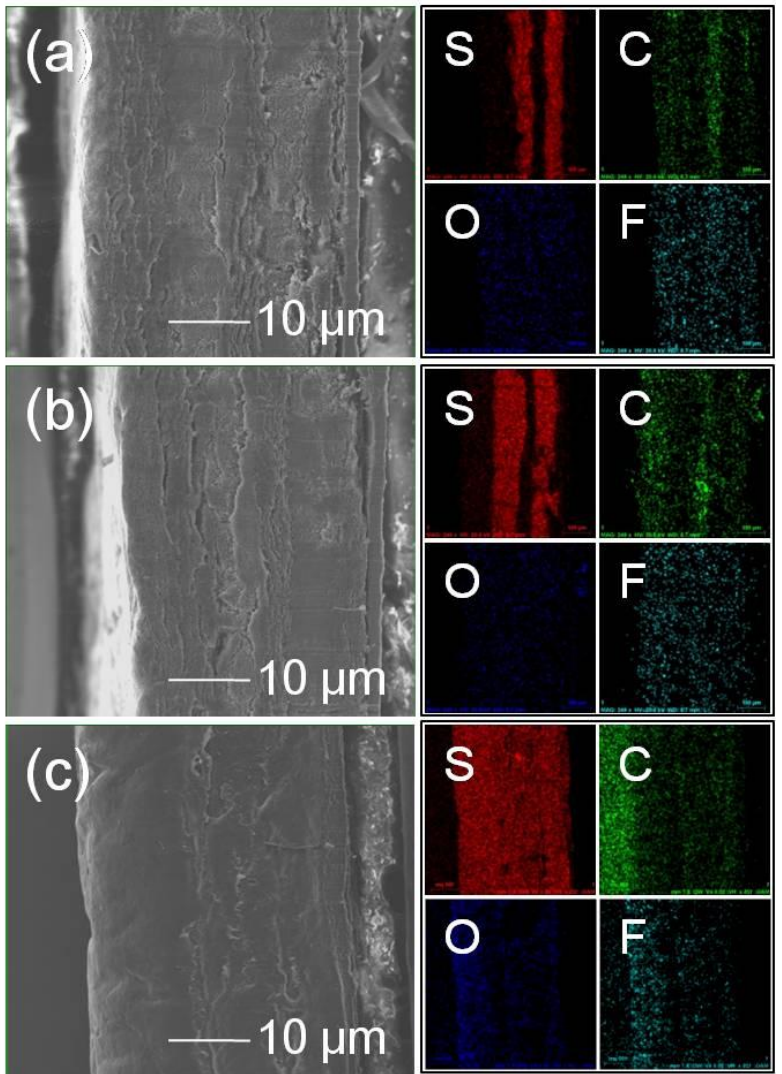

Figure 7 SEM/EDX inspection of the active-material layers after (a) 0, (b) 5, and (c) 200 cycles.

\subsection{Polysulfide-retention process of the hierarchical electrodes}

The important prerequisite for a favorable active-material rearrangement is superior polysulfide retention within the hierarchical electrode construction. On the contrary, an unfavorable active-material rearrangement incurs the detrimental polysulfide diffusion out from the cathode structure. Once escaped from the cathode region, dissolved polysulfides can quickly penetrate through the separator and either shuttle between the two electrodes or conglomerate into a shielding layer on the electrodes, blocking further use of lithium ions and active sulfur.

Therefore, in this section, a carbon-film layer is inserted in between the two layers of separators as a polysulfide trap for (i) a qualitative evaluation of the presence or absence of polysulfide diffusion and (ii) for the identification of the excellent polysulfide retention brought about by the hierarchical electrode. The polysulfide-trap cell configuration shown in 
Figure $8 \mathrm{a}$ is configured with a cathode, the first layer of a separator, a carbon-film layer (as the polysulfide trap), the second layer of a separator, and a lithium-foil anode. A reference polysulfide trap was inserted in a control cell using a conventional sulfur cathode with a sulfur loading of $2 \mathrm{mg} \mathrm{cm}^{-2}$. As a comparative analysis, experimental polysulfide traps equipped in a cell employing a hierarchical cathode with increasing sulfur loadings from 4.2 to $10.0 \mathrm{mg} \mathrm{cm}^{-}$ ${ }^{2}$ were sampled for exploring the polysulfide-retention process.

After cycling, the reference polysulfide trap shown in Figure $8 \mathrm{~b}$ is characterized by large domains of trapped active material and strong elemental sulfur signals as a result of severe polysulfide diffusion out from the conventional cathode. In contrast, the experimental polysulfide traps exhibit a well-maintained porous CNT-CNF network, as shown in Figures 8c $-8 f$. The SEM images reveal that only limited sulfur buildups were trapped by the cycled polysulfide trap so that the corresponding elemental mapping results exhibit weak sulfur signals. In view of the limited sulfur-containing species detected by the polysulfide trap, the SEM/EDX investigation into the polysulfide-trap cell demonstrates the effective polysulfideretention capability of the hierarchical electrode. In addition, as the active-material layers load with increasing amounts of sulfur, the cycled polysulfide traps still display smooth exterior with no surface coverings. This proves that the hierarchical electrode is successful in building high-loading cathodes with good polysulfide retention. As a result, the Li-S cells employing the high-loading hierarchical cathode demonstrate good long-term cycle stability.

A comparative analysis of these polysulfide traps shows solid evidence that severe polysulfide diffusion is suppressed by a well-designed structural cathode. Most importantly, the investigation of the battery chemistries explores the activation process of the activematerial layers and the polysulfide-retention process of the hierarchical electrodes, which allows us to draw this conclusion. The use of hierarchical cathodes stabilizes the dissolved polysulfide within the structural cathode region during electrochemical cycling. As a result, the hierarchical cathodes transform the dissolved polysulfides from the culprit in causing 
unstable Li-S battery chemistries to a favorable catholyte in facilitating efficient utilization and electrochemical stability of high-loading sulfur cathodes.
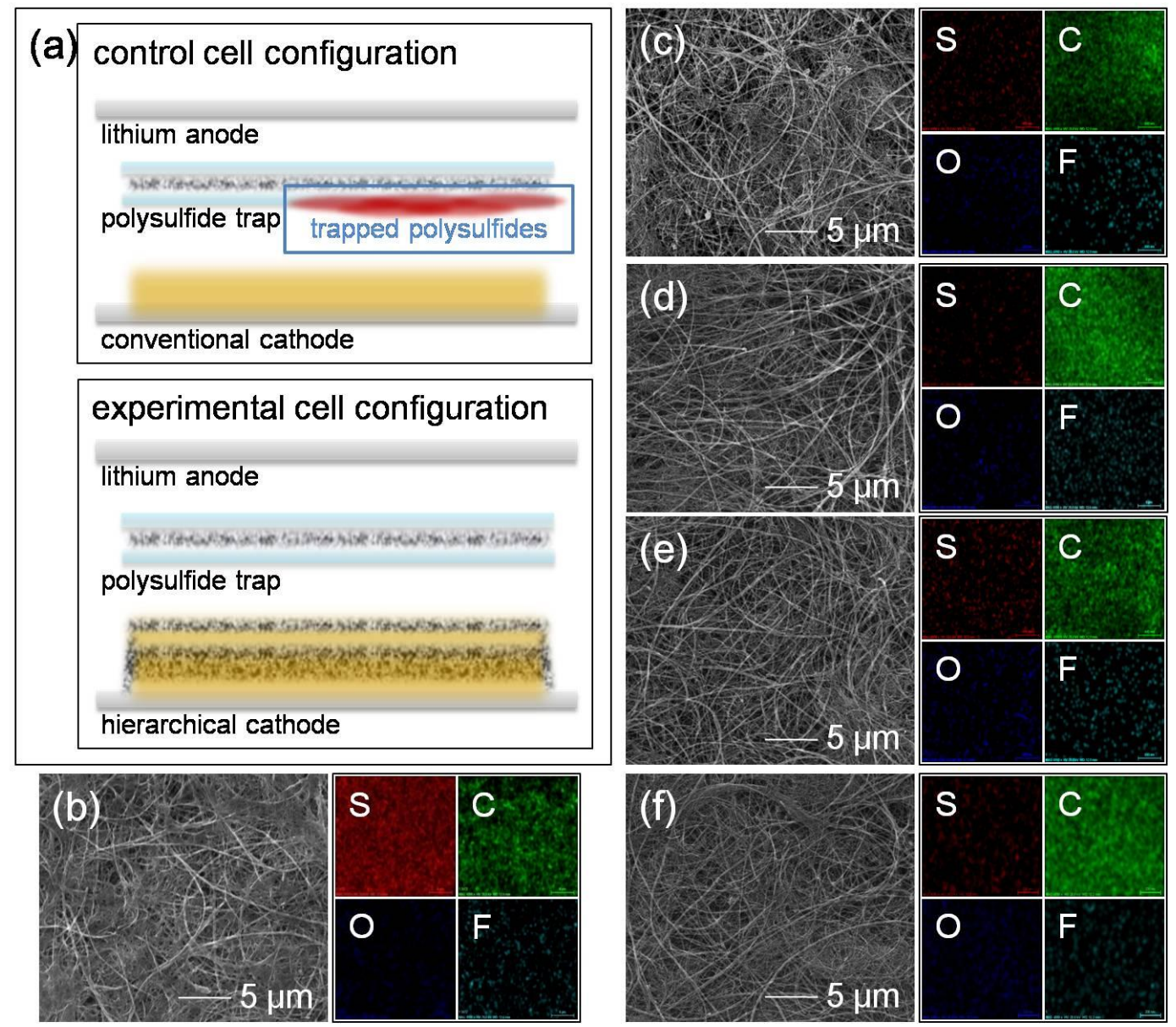

Figure 8 (a) polysulfide-trap configuration of a control cell employing the conventional cathode (upper) and an experimental cell employing the hierarchical cathode (lower). SEM/EDX inspection of the cycled polysulfide traps inserted in cells using (b) conventional cathodes and $(\mathrm{c}-\mathrm{f})$ the hierarchical cathodes with sulfur loadings of $4.2,6.3,8.3$, and 10.0 $\mathrm{mg} \mathrm{cm}{ }^{-2}$.

\section{Conclusion}

In summary, hierarchical cathodes develop high-loading cathodes with low capacityfade rates of $0.04-0.08 \%$ per cycle and long-term cyclability for $500-1,000$ cycles in Li-S cells. Benefiting from the stable electrochemical characteristics, the hierarchical cathodes serving as a testing platform demonstrate the potential for enhancing their sulfur loadings up to $10 \mathrm{mg} \mathrm{cm}^{-2}$ and implementing them in pouch cells. Finally, the cross-sectional microstructural inspection and the polysulfide-trap cells explore, respectively, the activation 
and the polysulfide-retention processes of the high-loading cathodes. The results demonstrate that the stable battery performances mainly result from the stabilization of dissolved polysulfides within the cathode region due to the strong tortuosity brought about by the hierarchical electrode configuration, benefiting the capacity retention. In addition, the dissolved polysulfides have high electrochemical activity, which activate the bulk sulfur clusters and enhance the redox-reaction capability of the high-loading sulfur cathode. Therefore, the utilization of dissolved polysulfides as electrochemically active catholyte in high-loading sulfur cathodes presents a potential opportunity to ameliorate the long-term cycle stability of the corresponding Li-S batteries.

\section{Acknowledgements}

This work was supported by the Assistant Secretary for Energy Efficiency and Renewable Energy, Office of Vehicle Technologies of the U.S. Department of Energy under Contract No. DE-EE007218.

\section{References}

[1] Y.-X. Yin, S. Xin, Y.-G. Guo, L.-J. Wan, Angew. Chem. Int. Ed. 52 (2013) 13186 13200.

[2] A. Manthiram, S.-H. Chung, C. Zu, Adv. Mater. 27 (2015) 1980 - 2006.

[3] S. Urbonaite, T. Poux, P. Novák, Adv. Energy Mater. 5 (2015) 1500118.

[4] R. D. Rauh, K. M. Abraham, G. F. Pearson, J. K. Surprenant, S. B. Brummer, J. Electrochem. Soc. 126 (1979) 523 - 527.

[5] E. Peled, Y. Sternberg, A. Gorenshtein, Y Lavi, J. Electrochem. Soc. 136 (1989) $1621-1625$.

[6] Y. V. Mikhaylik, J. R. Akridge, J. Electrochem. Soc. 151 (2004) A1969 - A1976.

[7] S.-E. Cheon, S.-S. Choi, J.-S. Han, Y.-S. Choi, B.-H. Jung, H. S. Lim J. Electrochem. Soc. 151 (2004) A2067 - A2073. 
[8] C. Barchasz, F. Molton, C. Dubo, J.-C. Leprêtre, S. Patoux, F. Alloin, Anal. Chem. 84 (2012) $3973-3980$.

[9] D. Bresser, S. Passerini, B. Scrosati, Chem. Commun. 49 (2013) 10545 - 10562.

[10] M. Hagen, D. Hanselmann, K. Ahlbrecht, R. Maça, D. Gerber, J, Tübke, Adv. Energy Mater. 5 (2015) 1401986.

[11] J. Xiao, Adv. Energy Mater. 5 (2015) 1501102.

[12] D.-W. Wang, Q. Zeng, G. Zhou, L. Yin, F. Li, H.-M. Cheng, I. R. Gentle, G. Q. Lu, J. Mater. Chem. A 1 (2013) 9382 - 9394.

[13] L. Chen, L. L. Shaw, J. Power Sources 267 (2014) 770 - 783.

[14] Y. Son, J.-S.Lee, Y. Son, J.-H. Jang, J. Cho, Adv. Energy Mater. 5 (2015) 201500110.

[15] M. A. Pope, I. A. Aksay, Adv. Energy Mater. 5 (2015) 201500124.

[16] L. Wang, Y. Zhao, M. L. Thomas, H. R. Byon, Adv. Funct. Mater. 24 (2014) 2248 2252.

[17] J. Song, T. Xu, M. L. Gordin, P. Zhu, D. Lv, Y.-B. Jiang, Y. Chen, Y. Duan, D. Wang, Adv. Funct. Mater. 24 (2014) 1243 - 1250.

[18] S. Thieme, J. Bruckner, I. Bauer, M. Oschatz, L. Borchardt, H. Althues, S. Kaskel, J. Mater. Chem. A 1 (2013) 9225 - 9234.

[19] C. Tang, Q. Zhang, M.-Q. Zhao, J.-Q. Huang, X.-B. Cheng, G.-L. Tian, H.-J. Peng, F. Wei, Adv. Mater. 26 (2014) $6100-6105$.

[20] M.-Q. Zhao, H.-J. Peng, G.-L. Tian, Q. Zhang, J.-Q. Huang, X.-B. Cheng, C. Tang, F. Wei, Adv. Mater. 26 (2014) $7051-7058$.

[21] Y. Zhou, C. Zhou, Q. Li, C. Yan, B. Han, K. Xia, Q. Gao, J. Wu, Adv. Mater. 27 (2015) $3774-3781$

[22] J. Wang, Y.-S. He, J. Yang, Adv. Mater. 27 (2015) 569 - 575.

[23] P. Strubel, S. Thieme, T. Bimelt, A. Helmer, M. Oschatz, J. Bruckner, H. Althues, S. Kaskel, Adv. Funct. Mater. 25 (2015) 287 - 297. 
[24] F. Wu, J. T. Lee, F. Fan, N. Nitta, H. Kim, T. Zhu, G. Yushin, Adv. Mater. 27 (2015) $5579-5586$.

[25] J. Shim, K. A. Striebel, E.J. Cairns, J. Electrochem. Soc. 140 (2002) A1321 - A1325.

[26] D. Lv, J. Zheng, Q. Li, X. Xie, S. Ferrara, Z. Nie, L. B. Mehdi, N. D. Browning, J.-G Zhang, G. L. Graff, J. Liu, J. Xiao, Adv. Energy Mater. 5 (2015) 1402290.

[27] X.-B. Cheng, J.-Q. Huang, Q. Zhangn, H.-J. Peng, M.-Qi. Zhao, F. Wei, Nano Energy $4(2014) 65-72$.

[28] S.-H. Chung, C.-H. Chang, A. Manthiram, Small 12 (2016) 939 - 950.

[29] X. Fang, H. Peng, Small 11 (2015)1488 - 1511.

[30] S. Zhang, K. Ueno, K. Dokko, M. Watanabe, Adv. Energy Mater. 5 (2015) 1500117.

[31] R. Elazari, G. Salitra, A. Garsuch, A. Panchenko, D. Aurbach, Adv. Mater. 23 (2011) $5641-5644$.

[32] C. Barchasz, F. Mesguich, J. Dijon, J.-C. Leprêtre, S. Patoux, F. Alloin, J. Power Sources $211(2012) 19-26$.

[33] S.-H. Chung, A. Manthiram, Adv. Mater. 26 (2014) 1360 - 1365.

[34] S.-H. Chung, A. Manthiram, ACS Sustain. Chem. Eng. 2 (2014) 2248 - 2252.

[35] X.-B. Cheng, H.-J. Peng, J.-Q. Huang, L. Zhu, S.-H. Yang, Y. Liu, H.-W. Zhang, W. Zhu, F. Wei, Q. Zhang, J. Power Sources 261 (2014) 264 - 270.

[36] G. Zhou, S. Pei, L. Li, D.-W. Wang, S. Wang, K. Huang, L.-C. Yin, F. Li, H.-M. Cheng, Adv. Mater. 26 (2014) $625-631$.

[37] S.-H. Chung, A. Manthiram, Chem. Commun. 50 (2014) $4184-4187$.

[38] S.-H. Chung, R. Singhal, V. Kalra, A. Manthiram, J. Phys. Chem. Lett. 6 (2015) 2163 2169.

[39] J. Balach, T. Jaumann, M. Klose, S. Oswald, J. Eckert, L. Giebeler, J. Phys. Chem. C 119 (2015) $4580-4587$. 
[40] G. Ma, Z. Wen, Q. Wang, C. Shen, P. Peng, J. Jin, X. Wu, J. Power Sources 273 (2015) $511-516$

[41] Z. Xiao, Z. Yang, L. Wang, H. Nie, M. Zhong, Q. Lai, X. Xu, L. Zhang, S. Huang, Adv. Mater. 27 (2015) $2891-2898$.

[42] S.-H. Chung, A. Manthiram, Adv. Funct. Mater. 24 (2014) 5299 - 5306.

[43] S.-H. Chung, A. Manthiram, Adv. Mater. 26 (2014) 7352 - 7357.

[44] S.-H. Chung, P, Han, R. Singhal, V. Kalra, A. Manthiram, Adv. Energy Mater. 5 (2015) 1500738

[45] J. Balach, T. Jaumann, M. Klose, S. Oswald, J. Eckert, L. Giebeler, Adv. Funct. Mater. $25(2015) 5285-5291$.

[46] C.-H. Chang, S.-H. Chung, A. Manthiram, J. Mater. Chem. A 3 (2015) 18829 - 18834.

[47] G. Wang, Y. Lai, Z. Zhang, J. Li, Z. Zhang, J. Mater. Chem. A 3 (2015) 7139 - 7144.

[48] C.-H. Chang, S.-H. Chung, A. Manthiram, Small 12 (2016) $174-179$.

[49] U. Stoeck, J. Balach, M. Klose, D. Wadewitz, E. Ahrens, J. Eckert, L. Giebeler, J. Power Sources 309 (2016) $76-81$.

[50] J.-Q. Huang, Q. Zhang, H.-J. Peng, X.-Y. Liu, W.-Z. Qian, F. Wei, Energy Environ. Sci. 7 (2014) $347-353$.

[51] N. Yan, X. Yang, W. Zhou, H. Zhang, X. Lia, H. Zhang, RSC Adv. 5 (2015) 26273 26280.

[52] J.-Q. Huang, Q. Zhang, F. Wei, Energy Storage Mater. 1 (2015) 127 - 145.

[53] J.Q. Huang, T.-Z. Zhuang, Q. Zhang, H.-J. Peng, C.-M. Chen, F. Wei, ACS Nano 9 (2015) $3002-3011$.

[54] J. He, Y. Chen, W. Lv, K. Wen, P. Li, F. Qi, Z. Wang, W. Zhang, Y. Li, W. Qin, W. He, J. Power Sources 327 (2016) $474-480$.

[55] J. He, Y. Chen, W. Lv, K. Wen, P. Li, Z. Wang, W. Zhang, W. Qin, W. He, ACS Energy Lett. 1 (2016) $16-20$. 
[56] F. Wu, J. T. Lee, N. Nitta, H. Kim, O. Borodin, G. Yushin, Adv. Mater. 27 (2015) $101-108$.

[57] R. Cao, W. Xu, D. Lv, J. Xiao, J.-G. Zhang, Adv. Energy Mater. 5 (2015) 1402273.

[58] L. X. Yuan, X. P. Qiu, L. Q. Chen, W. T. Zhu, J. Power Sources 189 (2009) 127 - 132.

[59] W. Ahn, K. B. Kim, K. N. Jung, K. H. Shin, C. S. Jin, J. Power Sources 202 (2012) $394-399$.

[60] S. Risse, S. Angioletti-Uberti, J. Dzubiella, M. Ballauff, J. Power Sources 267 (2014) $648-654$

[61] Y.-Z. Fu, Y.-S. Su, A. Manthiram, Angew. Chem. Int. Ed. 52 (2013) 6930 - 6935.

[62] C.-P. Yang, Y.-X. Yin, H. Ye, K.-C. Jiang, J. Zhang, Y.-G. Guo, ACS Appl. Mater. Interfaces 6 (2014) $8789-8795$.

[63] X. Ji, L.F. Nazar, J. Mater. Chem. 20 (2010) 9821 - 9826. 


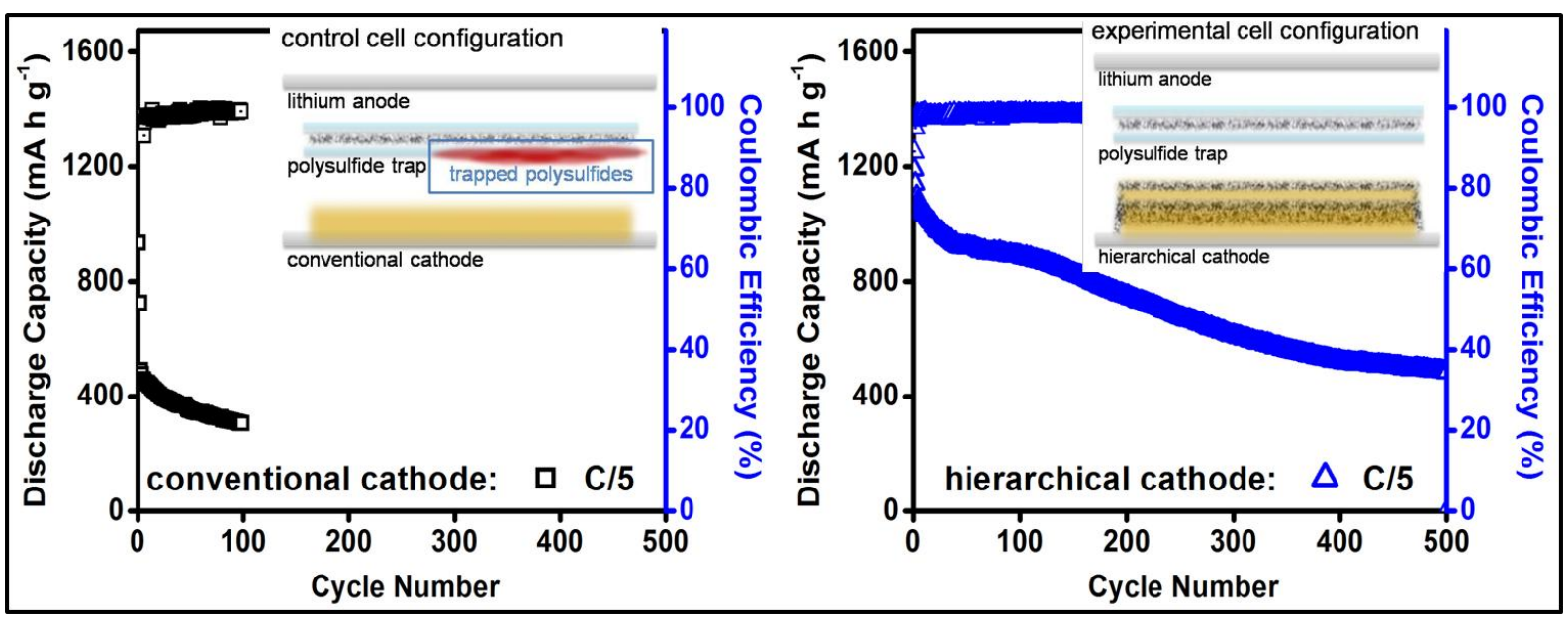

